\title{
BAOMS President welcomes independent review into diversity and inclusion
}

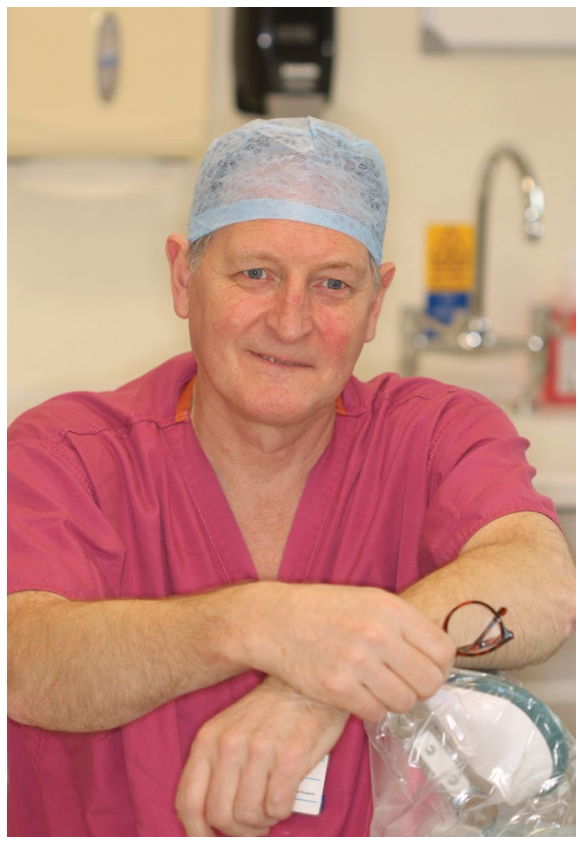

British Association of Oral and Maxillofacial Surgeons (BAOMS) President Austen Smith has welcomed the Royal College of Surgeons of England (RCSEng) independent review into diversity and inclusion, conducted by Dame Helena Kennedy QC.

Dr Smith (pictured) said: 'Given the impact of the RCSEng holding themselves up to a strong light in their report $A n$ independent review on diversity and inclusion for the Royal College of Surgeons of England:
An exciting call for radical change, ${ }^{1}$ it is clear that surgical disciplines in the UK owe it to present and future colleagues to squeeze out any unfairness or unacceptable labelling.

'As President of the British Association of Oral \& Maxillofacial Surgeons I am proud of what has already been achieved within our surgical specialty.

'From the initiation of the Inclusion, Equity and Diversity policy by my predecessor Sat Parmar in 2019, BAOMS has actively pursued a policy of cultivating an atmosphere that recognises and acknowledges the differences between individuals, without judgement.

'This has already resulted in the most diverse membership of BAOMS Council, which steers and guides our association. Half of Council members, for example, are women and from a range of different backgrounds.

'We have a publicly exhibited gallery on our website that illustrates the wide variety of origins, backgrounds, cultures and standpoints from junior doctors up to senior figures in the specialty.

'In 2023 our President will be Miss Daljit Kaur Dhariwal, our second female President who is of British Asian/Indian origin. Her Presidency will be clear proof that it is possible to have a satisfying family life, and still achieve pre-eminence in the demanding world of clinical surgery and medical politics.
'Council member Kanwalraj Moar, Cleft Oral Maxillofacial Surgeon and Divisional Director for Women's and Children's Services at Addenbrookes Hospital, has taken the BAOMS Diversity Lead portfolio. Her role is to monitor our diversity and inclusion activities, communicate our achievements and advise where we need to do better. In addition, she is a founding member and Honorary Secretary for the Society of Women in Maxillofacial Surgery (SWiMS), an independent society that in healthy collaboration with BAOMS represents the interests and mutual opinions of female surgeons active in our speciality.

'Oral and maxillofacial surgery is rich with a diversity of skills, characteristics and attributes that creates a vitality and strength. I believe that the diversity of our members and leaders is an amazing positive benefit to OMFS. And I hope those who gravitate to OMFS will feel and be both supported and protected as they pursue their career aspirations.'

\section{Reference}

1. Royal College of Surgeons of England. The Royal College - Our Professional Home. An independent review on diversity and inclusion for the Royal College of Surgeons of England. An exciting call for radical change. March 2021. Available at: https://www. rcseng.ac.uk/-/media/files/rcs/about-rcs/aboutour-mission/rcs-diversity-report--22-march-1.pd (accessed March 2021).

\section{Top five reasons for dental complaints during the coronavirus pandemic}

As the one-year anniversary of the UK's first lockdown due to the COVID-19 pandemic passes, the Dental Defence Union (DDU) has analysed the top five reasons for patients to raise complaints about pandemic dental practice.

The top five reasons for complaints were:

1. Treatment availability - delays caused by the closing of practices and the limitations on patient numbers after practices reopened

2. Dissatisfaction with treatment allegations of poor treatment outcomes and delayed diagnosis due to the pandemic

3. Fees and refund requests - patients requesting a refund or compensation for treatment delays or incomplete treatment

4. Communication issues - patients who felt they had not been properly informed about delays, cancellations and arrangements for urgent care during lockdown.

5. COVID-secure measures - issues such as infection control, PPE and the use of face masks.

The DDU supported dental practitioners with over 500 advisory and complaint cases between the start of lockdown in March to the end of 2020. In the majority of cases, members wanted advice on an aspect of patient care in order to preempt problems. Just $9 \%$ of cases were about complaints, and of these treatment availability was the most common reason for a patient to be dissatisfied.

However, the DDU says it anticipates that complaint numbers are likely to increase because of delays in treatment caused by the pandemic. 